\title{
RAMIFIED FREGE ARITHMETIC
}

\author{
RICHARD G HECK JR
}

\section{THE PROBLEM}

Frege's Theorem, in its most familiar form, states that second-order DedekindPeano arithmetic can be derived in second-order logic from Hume's Principle, or HP, using Frege's definitions of the basic arithmetical notions zero, predecession, and natural number. HP says that two 'concepts' have the same number if, and only if, they are in one-one correspondence. Formally:

$$
\begin{aligned}
N x: F x=N x: G x \text { iff } \exists R[ & \forall x \forall y \forall z \forall w(R x y \wedge R z w \rightarrow x=z \equiv y=w) \wedge \\
& \forall x(F x \rightarrow \exists y(R x y \wedge G y)) \wedge \\
& \forall y(G y \rightarrow \exists x(R x y \wedge F x))]
\end{aligned}
$$

Frege's definitions of zero, predecession, and natural number will be explained below. As for second-order Dedekind-Peano arithmetic, the axiomatization most convenient for our purposes is the following:

(1) $\mathbb{N} 0$

(2) $\mathbb{N} x \wedge P x y \rightarrow \mathbb{N} y$

(3) $\forall x \forall y \forall z(\mathbb{N} x \wedge P x y \wedge P x z \rightarrow y=z)$

(4) $\forall x \forall y \forall z(\mathbb{N} x \wedge \mathbb{N} y \wedge P x z \wedge P y z \rightarrow x=y)$

(5) $\neg \exists x(\mathbb{N} x \wedge P x 0)$

(6) $\forall x(\mathbb{N} x \rightarrow \exists y(P x y))$

(7) $\forall F(F 0 \wedge \forall x \forall y(\mathbb{N} x \wedge F x \wedge P x y \rightarrow F y) \rightarrow \forall x(\mathbb{N} x \rightarrow F x)$

If (slightly non-standardly) we take Frege arithmetic to be the second-order theory whose non-logical axioms are HP and Frege's definitions of the arithmetical notions, re-construed as axioms, then Frege's Theorem may

Thanks to John Burgess and Øystein Linnebo both for checking early versions of this proof and for the many helpful conversations we have had on this peculiar topic. Thanks, too, to an anonymous referee whose comments greatly simplified the proof of the main result and improved the exposition at several points.

This material was presented to a graduate seminar given at Harvard University in Fall 2004 and to a meeting of the Mathematics Workshop at Arché, the AHRC Research Centre for the Philosophy of Logic, Language, Mathematics and Mind at the University of St Andrews in February 2005. Thanks to Arché for its support, which is very much appreciated, and to Crispin Wright for arranging my visit. Thanks to everyone who attended these presentations for their comments and questions but especially to Stewart Shapiro for forcing several clarifications. 
be stated in the following form: Frege arithmetic extends Dedekind-Peano arithmetic. ${ }^{1}$

Frege's Theorem came to contemporary attention as a result of work by Crispin Wright, who presented a proof of it in Frege's Conception of Numbers as Objects [Wright, 1983]. Subsequent historical work has shown that a proof was also known to Frege himself [Heck, 1993, Boolos \& Heck, 1998]. Subsequent mathematical work has been driven by a desire to understand this surprising result: What is it about the seemingly innocent HP that gives it its strength? Precisely how do the axioms of arithmetic follow from it? In particular, how does it disgorge infinitely many natural numbers? Early work on this question was focused on HP itself. For example, George Boolos investigated the conditions HP imposes on the domain [Boolos, 1998c] and carefully analyzed the proof that every number has a successor [Boolos, 1998a]. Following his lead, I looked into the importance of the fact that HP, as it is usually formulated, assigns cardinal numbers to all concepts, including the universal concept, and so not just to finite ones [Heck, 1997a], and in other work discussed the significance of HP's impredicativity, that is, the fact that it treats numbers themselves as individuals, so that they are in the domain and range of some of the relations over which the quantifiers in HP range [Heck, 1997b]. There are, of course, philosophical questions that surround these mathematical ones, and there is now a substantial literature on them, too. ${ }^{2}$

More recently, attention has been focused on the logic used to derive the axioms of arithmetic from HP, the obvious question to ask being how weak that logic may be taken to be. The power of standard second-order logic derives from the so-called comprehension axioms, which are of the form

$$
\exists F \forall x[F x \equiv A(x)],
$$

\footnotetext{
${ }^{1}$ Frege arithmetic is usually taken to be simply second-order logic plus HP. There are two reasons for the change of terminology. First, and most importantly, the problem I intend to discuss has been solved for that case: John Burgess has shown that Robinson arithmetic can be interpreted in predicative second-order logic plus HP, but Burgess's proof uses a definition of natural number very different from Frege's [Burgess, 2005, pp. 1137]. The second reason is that, to a significant extent, the philosophical significance fans of Frege's Theorem have hoped it would have depends upon the use of Frege's definitions. The hope is that Frege's Theorem reveals something important about arithmetic as we ordinarily understand it. If so, however, the formal theorems derived from HP must express (something not too different from) what the corresponding arithmetical claims express. Hence, the definitions of central notions must reflect the ordinary meaning of those notions, and Frege's definitions are intended to do so.

Obviously these two reasons are connected: As Burgess himself notes, fans of Frege's Theorem won't find much of philosophical significance in his result.

${ }^{2}$ Reasonable starting points for anyone interested in those questions are [Demopoulos, 1995] and [?].
} 
each essentially asserting that a given formula $A(x)$ defines a 'concept', that is, something in the range of the second-order variables. Sub-systems of second-order logic arise from restrictions on comprehension, that is, on what sort of formula $A(x)$ may be. If, for example, we require it not to contain bound second-order quantifiers, we have predicative second-order logic. If we require it to be $\Pi_{1}^{1},{ }^{3}$ we have second-order logic with just $\Pi_{1}^{1}$ comprehension. And so forth. Full second-order logic, with unrestricted comprehension, gives rise to what we might call 'full' Frege arithmetic.

The question of interest may then be stated roughly as follows: What is the weakest natural fragment of Frege arithmetic in which Frege's Theorem can be proven? This rough statement of the question needs refinement, however. ${ }^{4}$ Frege's Theorem, as it is usually stated, is that full second-order arithmetic can be interpreted in second-order logic plus HP, using Frege's definitions. But it is easy to see that, if we define the concept of natural number in Frege's way, we shall never get any more induction than we are prepared to buy with comprehension axioms: If we assume just $\Pi_{1}^{1}$ comprehension, we will get just $\Pi_{1}^{1}$ induction, and so forth. So another way to put the question would be: What is the weakest fragment of Frege arithmetic in which a reasonable amount of arithmetic can be developed? That will depend, obviously, upon what one means by "a reasonable amount of arithmetic".

Careful examination of standard proofs of Frege's Theorem shows that no more than $\Pi_{1}^{1}$ comprehension is needed in them. ${ }^{5}$ Axioms (1) and (2) follow directly from Frege's definitions of zero, predecession, and natural number, with no appeal either to comprehension or to HP. Axioms (3), (4), and (5) can be derived from HP using only predicative comprehension. The proof of axiom (6), on the other hand, which asserts the existence of successor, appeals to $\Pi_{1}^{1}$ comprehension at several points, and $\varnothing$ ystein Linnebo has shown that axiom (6) cannot be proven in the predicative fragment of Frege arithmetic: There is a model of predicative Frege arithmetic in which axiom (6) is false [Linnebo, 2004]. ${ }^{6}$

\footnotetext{
${ }^{3}$ That is, of the form: $\forall F \ldots \forall G \phi$, where $\phi$ contains no second-order quantifiers, but may contain free second-order variables.

${ }^{4}$ Moreover, there are many other sorts of fragments of second-order logic, determined not only by other sorts of restrictions on comprehension but by other sorts of axioms that may be assumed, such as choice principles of various sorts. These are not always comparable in strength, so it may be that there is no single weakest such fragment. If not, then not.

${ }^{5}$ This observation was first made in [Heck, 2000, p. 192]. See [Linnebo, 2004, pp. 161ff] for the details. Linnebo also proves a converse: $\Pi_{1}^{1}$-FA can be interpreted in $\Pi_{1}^{1}$-PA.

${ }^{6}$ Here again I am assuming that 'Frege arithmetic' includes Frege's definitions of zero, predecession, and natural number. As noted above, Robinson arithmetic can be interpreted
} 
Linnebo also notes that $\Pi_{1}^{1}$ comprehension is needed for the usual proofs of the axioms governing addition and multiplication:

$$
\begin{aligned}
& x+0=x \\
& x+S y=S(x+y) \\
& x \times 0=0 \\
& x \times S y=(x \times y)+x
\end{aligned}
$$

No such axioms were included in the above axiomatization of second-order arithmetic, because they did not need to be: As Dedekind showed, we can define addition and multiplication in terms of zero and succession and then prove the axioms for addition and multiplication from those for zero and successor [Dedekind, 1902]. ${ }^{7}$ But those proofs-in particular, the proofs that sums and products are unique and always exist - are by induction, and the predicates with respect to which these inductions are carried out will of course contain the formulae that define addition and multiplication. Those formulae are $\Pi_{1}^{1}$, so $\Pi_{1}^{1}$ comprehension is needed for these proofs.

Finally, $\Pi_{1}^{1}$ comprehension is needed for the proof of axiom (7), that is, for the proof of mathematical induction. The point is easy to overlook. I have often said in lectures on Frege, and may even have said in print, that Frege defines the natural numbers as "the numbers for which induction works". But that is not quite right, as a close look at how Frege actually defines the natural numbers reveals. Frege defines the 'strong' ancestral of a relation $R$ as follows:

$$
R^{*} a b \stackrel{d f}{\equiv} \forall F[\forall x(R a x \rightarrow F x) \wedge \forall x \forall y(F x \wedge R x y \rightarrow F y) \rightarrow F b],
$$

and the 'weak' ancestral as:

$$
R^{*=} a b \stackrel{d f}{=} R^{*} a b \vee a=b .
$$

It is then easy to prove, via predicative comprehension, that:

$$
R^{*=} a b \equiv \forall F[F a \wedge \forall x \forall y(F x \wedge R x y \rightarrow F y) \rightarrow F b] .
$$

Frege then defines the concept of natural number as:

$$
\mathbb{N} n \stackrel{d f}{=} P^{*=} 0 n,
$$

where the relation $P$ is that of predecession, defined in a way we shall see shortly. We then have immediately that

$$
\mathbb{N} n \rightarrow \forall F[F 0 \wedge \forall x \forall y(F x \wedge P x y \rightarrow F y) \rightarrow F n],
$$

in predicative second-order logic plus HP, but the proof uses definitions other than Frege's, so there is no conflict with Linnebo's result.

${ }^{7}$ See [Heck, 1995] for a discussion of related results from Frege's Grundgesetze [?]. 
which is equivalent to:

$$
\forall F[F 0 \wedge \forall x \forall y(F x \wedge P x y \rightarrow F y) \rightarrow \forall x(\mathbb{N} x \rightarrow F x)] .
$$

But this is weaker than (7), due to the absence of $\mathbb{N} x$ from the antecedent of $F x \wedge P x y \rightarrow F y$. Now if we instantiate $F$ with $\mathbb{N} \xi \wedge F x$, thus arriving at:

$\forall F\{(\mathbb{N} 0 \wedge F 0) \wedge \forall x \forall y[(\mathbb{N} x \wedge F x) \wedge P x y \rightarrow(\mathbb{N} y \wedge F y)] \rightarrow \forall x[\mathbb{N} x \rightarrow(\mathbb{N} x \wedge F x)]\}$

then (7) will follow quickly, but this move depends upon comprehension, specifically upon the availability of

$$
\exists G \forall x[G x \equiv \mathbb{N} x \wedge F x],
$$

and the definition of $\mathbb{N}$ contains bound second-order quantifiers: It is, more precisely, $\Pi_{1}^{1}$. So $\Pi_{1}^{1}$ comprehension is sufficient here, but it also seems to be necessary.

So second-order arithmetic with $\Pi_{1}^{1}$ induction $^{8}$ can be interpreted in secondorder arithmetic with $\Pi_{1}^{1}$ comprehension. Can this result be improved? We have seen that there are three places $\Pi_{1}^{1}$ comprehension is needed in the usual proofs of Frege's Theorem: In the proof that every number has a successor, in the proof of the axioms for addition and multiplication, and in the proof of induction. Even if our goal were just to interpret first-order arithmetic, all of these would remain obstacles.

As it happens, two of these obstacles can be overcome. First, Linnebo's work leaves open the possibility that the existence of successor can be proven in ramified predicative second-order logic. And, perhaps surprisingly, it can be. That is the main technical result of this note.

Second, work by John Burgess and Allen Hazen [Burgess \& Hazen, 1998] implies that, if we can prove the existence of successor, then we can define addition and multiplication and prove the axioms that govern them. More precisely, consider what we might call the simple theory of successor, the theory whose axioms are the universal closures of

(1) $0 \neq S x$

(2) $S x=S y \rightarrow x=y$

(3) $x \neq 0 \rightarrow \exists y(x=S y)$

It is well known that the first-order version of this theory is decidable. What Burgess and Hazen show, however, is that, if the logic of the theory is taken to be ramified predicative second-order logic, then this theory interprets

\footnotetext{
${ }^{8}$ Again, axiom (7) will give us only as much induction as we buy with comprehension.
} 
$I \Delta_{0}(\exp ) .{ }^{9}$ That's not everything, but it's a significant amount of arithmetic. Alternatively - and, from the present point of view, perhaps more interestingly, because these definitions are more in the spirit of Frege's own definitions - one can define addition and multiplication as cardinal addition (roughly, as disjoint union) and cardinal multiplication (roughly, as the cardinality of the Cartesian product) and then interpret the usual axioms governing addition and multiplication using techniques Burgess adapts from earlier work on weak fragments of arithmetic. ${ }^{10}$ That will be enough to allow Robinson arithmetic, or Q ${ }^{11}$ to be interpreted in ramified Frege arithmetic. Results due to Edward Nelson and Alex Wilkie then show that $I \Delta_{0}$ and even slightly stronger theories can be interpreted, as well. Again, that's not everything, but it's a significant amount of arithmetic. ${ }^{12}$

There are probably limits to how far we can go in this direction: It does not seem likely that full first-order arithmetic is interpretable in ramified Frege arithmetic. ${ }^{13}$ But the interesting point that emerges is why not. If you had asked me not long ago, I would have told you that it was because Frege's proof that every number has a successor makes essential use of impredicative second-order reasoning. Well, I was wrong. Frege's proof does make use of impredicative second-order reasoning, but impredicative reasoning is not, in fact, essential to Frege's proof. The proof I shall give below follows Frege's quite closely: Modulo the need to prove and use

\footnotetext{
${ }^{9} I \Delta_{0}$, to be mentioned shortly, is PA with induction limited to $\Delta_{0}$ formulae, that is, formulae in which only 'bounded' quantifiers—quantifiers of the forms $\forall x<y$ and $\exists x<$ $y$-occur. (In such theories, we take $<$ as a primitive subject to the axiom that is otherwise used to define it: $x<y \equiv \exists z(y=x+S z)$.)

Exponentiation can be defined by a $\Delta_{0}$ formula, but $I \Delta_{0}$ does not prove that exponentiation is total. So $I \Delta_{0}(\exp )$ adds a function-symbol for exponentation and adds the recursion equations for it as axioms: $\exp (0)=1 ; \exp (S n)=2 \times \exp (n)$.

${ }^{10}$ This observation is due to John Burgess. Showing that sums and products are unique and that the usual axioms are satisfied when sums and products exist is a simple matter. It is the proof that sums and products exist that is difficult. But it turns out to be sufficient to show that the predicates " $\forall n(\xi+n$ exists and is unique)" and " $\forall n(\xi \times n$ exists and is unique)" are inductive. One then uses these formulae to re-define what counts as a natural number: Natural numbers are those things for which sums and products always exist and are unique. (That makes it sound easier than it is, but that is the idea.) See [Burgess, 2005] for the details. It appears that this can be extended to cardinal exponentiation, as well.

It does have to be admitted that, in defining addition and multiplication in this way, we are modifying the definition of 'natural number', whence the question how well our definition captures the ordinary meaning of this notion can again be raised. It's a nice question whether there is a way around this, say, by going up a few more degrees.

${ }^{11}$ The axioms of $\mathrm{Q}$ are those of the simple theory of successor plus the recursion equations for addition and multiplication.

${ }^{12}$ For some of the details of the work mentioned, see [Hájek \& Pudlák, 1993].

${ }^{13}$ See note 14.
} 
what amounts to a restricted form of reducibility, Lemma 13, my proof just is Frege's proof.

The lesson is thus that impredicative second-order reasoning is needed for the proof of Frege's Theorem only in so far as it is needed for the proof of mathematical induction. But it was only to be expected that it would be needed there: As said before, it's been obvious for some time that we will only get as much induction as we're prepared to buy with comprehension.

The remainder of the paper is devoted to the proof that the existence of successor can be proven in ramified Frege arithmetic. Section 2 describes the system itself and considers how HP and Frege's definitions of arithmetical notions should be formalized within it. Section 3 analyzes the usual proof that successors exist to identify where, precisely, impredicative comprehension is used. Section 4 explores the question how we can work around these uses of impredicative comprehension. Section 5 contains the actual proof that every number has a successor.

\section{RAMIFIED Frege ARITHMETIC}

In ramified second-order logic, predicate variables are divided into degrees, which I shall indicate by subscripts on such variables. So, for example, $F_{0}$ is a predicate variable of degree 0 , and $R_{3}$ is a predicate variable of degree 3. Comprehension takes the form:

$$
\exists F_{n} \forall x\left[F_{n} x \equiv A(x)\right],
$$

where $A(x)$ is a formula containing no bound second-order variables of degree higher than $n-1$, and no free second-order variables of degree higher than $n$. I shall use similar notation to indicate the degree of defined notions.

In such a language, HP admits of a wide variety of formulations. The first question is what degree we should assign the relation variable occuring on the right-hand side of HP. ${ }^{14}$ For present purposes, we can take it to be of

\footnotetext{
${ }^{14}$ Actually, the first question, in any context in which we do not have full comprehension, is how to formalize the cardinality operator. One may regard it as a functor, attaching only to variables-it might then be written: $\# F_{n}$ - or one may regard it as a term-forming operator, attaching to arbitrary formulae, in which case it would be written: $N x: A(x)$. The former gives rise to a system that could prove weaker. Burgess has given a constructive consistency proof for systems with a functor \#. It follows that we cannot interpret $I \Sigma_{1}$ in ramified Frege arithmetic, formulated with \#. (In fact, we cannot get nearly that much: See [Burgess, 1998] and [Burgess, 2005, pp. 128ff].) It is not known whether treating ' $N$ ' as a variable-binding term-forming operator, attaching to arbitrary formulae, increases the strength of such systems, but it now seems likely to many of us that it does. How much is unclear.

As it happens, this choice does not affect anything we shall do here. So I shall use the operator $N x: A(x)$, as it allows for cleaner formulations, and make appropriate remarks about the operator version in the notes.
} 
lowest degree, so that HP takes the form: ${ }^{15}$

$$
\begin{aligned}
N x: A(x)=N x: B(x) \text { iff } \exists R_{0}[ & \forall x \forall y \forall z \forall w\left(R_{0} x y \wedge R_{0} z w \rightarrow x=z \equiv y=w\right) \wedge \\
& \forall x\left(A(x) \rightarrow \exists y\left(R_{0} x y \wedge B(y)\right)\right) \wedge \\
& \left.\forall y\left(B(y) \rightarrow \exists x\left(R_{0} x y \wedge A(x)\right)\right)\right]
\end{aligned}
$$

Note that $A$ and $B$ are syntactic variables, ranging over arbitrary formulae. It may be worth exploring the relation between this form of $\mathrm{HP}$ and the other forms that arise when one takes $R$ to be of other degress. I will not undertake that investigation here, however.

As noted above, axioms (1), (2), (3), (4), and (5) of Dedekind-Peano arithmetic are all provable in predicative Frege arithmetic, using Frege's definitions. Something similar will obviously be true in the case of ramified Frege arithmetic, though we first have to make some choices about how to formulate Frege's definitions of arithmetical notions. Frege's definition of zero- $0=N x: x \neq x$-does not pose any problem, since no secondorder quantifiers occur in it. Such quantifiers do occur, however, in Frege's definitions of $P$ and $\mathbb{N}$, so we must decide what degrees to assign to the bound predicate variables appearing in those definitions.

2.1. Predecession. Frege's definition of predecession, as formulated in standard second-order logic, is:

$$
P m n \equiv \exists F \exists y[n=N x: F x \wedge F y \wedge m=N x:(F x \wedge x \neq y)]
$$

The question is what degree we should assign to the predicate variable $F$. For our purposes here, we may simply assign it degree zero, so that the definition becomes: ${ }^{16}$

$$
P_{1} m n \equiv \exists F_{0} \exists y\left[n=N x: F_{0} x \wedge F_{0} y \wedge m=N x:\left(F_{0} x \wedge x \neq y\right)\right]
$$

It is nonetheless worth considering the question how we should define predecession more generally, as it may be of some independent interest. Consider the definition schema:

$$
P_{k+1} m n \equiv \exists F_{k} \exists y\left[n=N x: F_{k} x \wedge F_{k} y \wedge m=N x:\left(F_{k} x \wedge x \neq y\right)\right]
$$

Here the subscript on the defined term $P$ indicate the degree of the formula that defines it, which is one greater than the degree of any bound secondorder variable occurring in that formula. That is, we have, in general:

$$
\exists R_{k+1} \forall x \forall y\left[R_{k+1} x y \equiv P_{k+1} x y\right],
$$

\footnotetext{
${ }^{15}$ In the functor formulation, we of course have $\# F_{0}=\# G_{0}$ iff $\ldots$, but HP is otherwise unchanged.

${ }^{16}$ If we want to use the functor $\# F_{n}$, then the definition must be given as:

$$
P_{1} m n \equiv \exists F_{0} \exists G_{0} \exists y\left[\forall x\left(F_{0} x \equiv G_{0} x \wedge x \neq y\right) \wedge n=\# G_{0} \wedge m=\# F_{0}\right] .
$$
}

Note that $P_{1}$ is still of degree 1 , so nothing significant changes below. 
so we may treat $P_{k+1}$ as if it were a free variable of degree $k+1$.

It is not difficult to see, by reflecting on the model Linnebo produces to show that axiom (6) is not provable in simple predicative second-order logic, that the various $P_{k}$ will typically have different extensions. However, although their extensions may differ, they will never conflict. It is obvious, in particular, that: $P_{k} m n \rightarrow P_{k+l} m n$, the reason being that any concept of degree $k$ is also of degree $k+l .{ }^{17}$ The converse is not provable, in general, but a restricted form is provable. Say that $n$ is a number of degree $k$ if it is the number of some set of degree $k-1$ :

$$
\operatorname{Num}_{k}(n) \equiv \exists F_{k-1}\left(n=N x: F_{k-1} x\right)
$$

Then the restriction we need is that $n$ is a number of degree $k$ :

Remark 1. $\operatorname{Num}_{k}(n) \rightarrow P_{k} m n \equiv P_{k+l} m n$

Proof. As noted earlier, the left-to-right direction is trivial. So we must prove that, if $\exists F_{k-1}\left(n=N x: F_{k-1} x\right)$ and $P_{k+l} m n$, then $P_{k} m n$. So suppose that $n=N x: F_{k-1} x$ and suppose that $P_{k+l} m n$. Let $j=k+l-1$. By the definition of $P_{k+l}$, there are $G_{j}$ and $b$ such that $G_{j} b, n=N x: G_{j} x$, and $m=N x:\left(G_{j} x \wedge x \neq b\right)$. So $N x: F_{k-1} x=N x: G_{j} x$, and so, by HP, there is a 1-1 relation $R_{0}{ }^{18}$ that correlates the $G_{j} \mathrm{~s}$ with the $F_{k-1} \mathrm{~s}$, and so for some $c$, $R_{0} b c$ and $F_{k-1} c$. Now, by the definition of $P_{k}$ :

$$
P_{k} m n \text { iff } \exists F_{k-1} \exists z\left[n=N x: F_{k-1} x \wedge F_{k-1} z \wedge m=N x:\left(F_{k-1} x \wedge x \neq z\right)\right]
$$

We need only show that $m=N x:\left(F_{k-1} x \wedge x \neq c\right)$. But $R_{0} \xi \eta$ also correlates the $G_{j}$ s other than $b$ with the $F_{k-1}$ s other than $c$. Hence, $N x:\left(G_{j} x \wedge x \neq\right.$ $b)=N x:\left(F_{k-1} x \wedge x \neq c\right)$. Since $m=N x:\left(G_{j} x \wedge x \neq b\right)$, we are done.

My own early investigations of the matters under discussion here made use of these various versions of predecession and the relation just proved to hold between them. As it happens, I eventually stumbled upon a cleaner approach. But the Remark may yet prove of use, and it is a simple example of a phenomenon that will be important later.

Henceforth, I write $P_{1} m n$ as just: $P m n$, dropping the subscript to avoid cluttering the formulae.

Given this definition of predecession, the usual proofs of axioms (3), (4), and (5) can then easily be transcribed into the present framework. For example, suppose that $P y 0$. Then, for some $F_{0}$ and for some $a$, we have: $N x: F_{0} x=0, F_{0} a$, and $N x:\left(F_{0} x \wedge x \neq a\right)=y$. And so, by HP, there is a

\footnotetext{
${ }^{17}$ That is, we always have: $\exists F_{k+l} \forall x\left[F_{k+l} x \equiv F_{k} x\right]$, as an instance of comprehension.

${ }^{18}$ Note that the proof here does not depend significantly upon the decision we made above regarding the degree of this variable.
} 
relation $R_{0}$ that correlates the $F_{0}$ s one-one with the non-self-identicals. But since $F_{0} a$, that is impossible.

This proof actually delivers something stronger than (5), namely:

$$
5^{\prime} . \neg \exists x(P x 0)
$$

The increased strength is a result of the fact that the condition $\mathbb{N} x$ that is present in (5) has vanished. Frege's proofs also deliver strengthened versions of (3) and (4):

$$
\begin{aligned}
& 3^{\prime} . \quad \forall x \forall y \forall z(P x y \wedge P x z \rightarrow y=z) \\
& 4^{\prime} . \quad \forall x \forall y \forall z(P x z \wedge P y z \rightarrow x=y)
\end{aligned}
$$

This fact will be crucial below.

2.2. Natural Number. Frege's definition of natural number famously depends upon his definition of the ancestral. In this case, too, we can give the definition at any degree we like and therefore have a family of similar notions. We define $*^{k}$ as an operator on formulae that binds two variables. So, where $A(x, y)$ is a formula, we have:

$$
\begin{gathered}
*_{x y}^{k}(A(x, y), a, b) \equiv \\
\forall F_{k-1}\left[\forall x\left(A(a, x) \rightarrow F_{k-1} x\right) \wedge \forall x \forall y\left(F_{k-1} x \wedge A(x, y) \rightarrow F_{k-1} y\right) \rightarrow F_{k-1} b\right] .
\end{gathered}
$$

In principle, $A(x, y)$ can be of any degree, but it is convenient to require that it be of degree $\leq k$ : that is, that it contain no free variables of degree greater than $k$ and no bound variables of degree greater than $k-1$. In that case, the degree of the defined notion will be $k$, as indicated by the superscript. Henceforth, I shall write $R_{k}^{*} a b$ instead of using the official, more cumbersome notation, with the understanding, again, that Rxy is a formula of degree no greater than $k$.

One quickly sees that $R_{k+l}^{*} a b \rightarrow R_{k}^{*} a b$, again because every concept of degree $k$ is a set of degree $k+l$. The converse is not provable. ${ }^{19}$

We define the weak ancestrals as follows:

$$
R_{k}^{*=} a b \equiv R_{k}^{*} a b \vee a=b
$$

Our attention here will be focused upon the case where $R x y$ is $P x y$. In that case, we will write, instead of ' $P_{k}^{*} a b$ ', just: $a<_{k} b$, and similarly: $a \leq_{k} b$. $^{20}$

\footnotetext{
${ }^{19}$ In an earlier verison of the proof to be given below, I relied upon the following partial converse: If $k \geq 2$, then $\left(4^{\prime}\right),\left(5^{\prime}\right) \vdash 0 \leq_{k+2} n \rightarrow \forall x\left(x<_{k} n \rightarrow x<_{k+1} n\right)$. As it happens, a simplification made possible by an observation by an anonymous referee-see note 25 made appeal to this result unnecessary. But it is perhaps worth mentioning, anyway.

${ }^{20}$ Note that, since $P x y$ is of degree 1 - it contains only a bound second-order variable of degree zero- $P_{k}^{*} a b$ is indeed of degree $k$ and so the notation $a<_{k} b$ is not misleading as regards the subscript. (It can be misleading in a different way, since we can have $a<_{k} a$.)
} 
We thus have a sequence of definitions of natural number as well:

$$
\mathbb{N}_{k} x \equiv 0 \leq_{k} x
$$

with the first of these being $\mathbb{N}_{1}$. As noted, if $k \leq l$, then $\mathbb{N}_{l} x \rightarrow \mathbb{N}_{k} x$, but the converse does not, in general, hold. For the most part, the ' $\mathbb{N}_{k} x$ ' will not often appear in what follows: ' $0 \leq_{k} x$ ' is already sufficiently terse, and the notational complexities are already daunting.

\section{The Usual Proof of the Existence of Successor}

One of the most surprising discoveries yet made about the proof of Frege's theorem is due to George Boolos: In full Frege arithmetic, axiom (6)which asserts the existence of successors-is redundant [Boolos, 1998c]. More precisely, consider the following principle, which asserts the extensionality of the cardinality operator $N:^{21}$

$$
\forall x[A(x) \equiv B(x)] \rightarrow N x: A(x)=N x: B(x)
$$

Boolos calls this principle 'Log', on the ground that it is a logical truth: It is true in every model, since models are extensional. Boolos's observation was that axiom (6) can be derived, in standard second-order logic, from Frege's definitions of the arithmetical notions, axiom (3), and Log. In particular, no appeal to HP is needed. Careful examination of Frege's own proof of axiom (6) shows, in fact, that he himself derives it from axioms (3), (4), and (5), and Log. It is this weaker claim that we shall be exploiting: What we shall show is that, in ramified second-order logic, axiom (6) follows from Log, the versions of Frege's definitions mentioned in the previous section, and $\left(3^{\prime}\right),\left(4^{\prime}\right)$, and $\left(5^{\prime}\right) .{ }^{22}$

The object is thus to adapt Frege's proof of axiom (6) in full Frege arithmetic to ramified Frege arithmetic. It will thus be worth our having a version of Frege's proof before us.

Lemma 2. (Grundgesetze 145) $0 \leq n \rightarrow \neg n<n$

Proof. The proof is by induction on the predicate: $\neg \xi<\xi$. So we must show: ${ }^{23}$

${ }^{21}$ In its functor form, $\log$ is: $\forall x\left(F_{m} x \equiv G_{n} x\right) \rightarrow \# F_{m}=\# G_{n}$.

${ }^{22}$ So far as I can see, axiom (6) will not follow just from axiom $\left(3^{\prime}\right)$, since $\left(4^{\prime}\right)$ and $\left(5^{\prime}\right)$ play important roles in the proof to be given below. This probably reflects the fact that, in the derivation of (6) from (3), we have to load a large number of conditions into the predicates over which the various inductions are performed. That does not require us to use anything stronger than $\Pi_{1}^{1}$ comprehension, but it does seem to make the use of $\Pi_{1}^{1}$ comprehension harder to avoid.

${ }^{23}$ The form of induction being used here is licensed by:

$$
R^{*=} a b \rightarrow \forall F[F a \wedge \forall x \forall y(F x \wedge R x y \rightarrow F y) \rightarrow F b],
$$




$$
\begin{aligned}
& \text { (i) } \neg 0<0 \\
& \text { (ii) } \neg m<m \wedge P m n \rightarrow \neg n<n
\end{aligned}
$$

For (i), suppose $0<0$. We then have what Boolos called the 'roll-back theorem':

$$
R^{*} a b \rightarrow \exists x\left(R x b \wedge R^{*=} a x\right)
$$

And so, in this case, for some $x, P x 0$ and $0 \leq x$. But that contradicts axiom (5).

For (ii), suppose that Pmn and suppose that $n<n$. By the roll-back theorem, again, for some $y, P y n$ and $n \leq y$. Since Pmn, axiom (4) implies that $y=m$. But then, by the transitivity of the ancestral, $m<m$, and we are done.

Lemma 3. (Grundgesetze 102) $F y \rightarrow P(N x:(F x \wedge x \neq y), N x: F x)$

Proof. By definition, $P(N x:(F x \wedge x \neq y), N x: F x)$ if, for some $G$ and some $z, G z, N x:(F x \wedge x \neq y)=N x:(G x \wedge x \neq z)$, and $N x: F x=N x: G x$. But we may obviously just take $G$ to be $F$ and $z$ to be $y$.

Theorem 4. (Grundgesetze 155) $0 \leq n \rightarrow P(n, N x: x \leq n)$

Proof. The proof is by induction on the predicate: $P(\xi, N x: x \leq \xi)$, the induction being of the strong form:

$$
0 \leq n \wedge F 0 \wedge \forall x \forall y(0 \leq x \wedge F x \wedge P x y \rightarrow F y) \rightarrow F n
$$

So we must show:

(i) $P(0, N x: x \leq 0)$

(ii) $0 \leq m \wedge P(m, N x: x \leq m) \wedge P m n \rightarrow P(n, N x: x \leq n)$

For (i), by Grundgesetze 102, $P(N x:(x \leq 0 \wedge x \neq 0), N x: x \leq 0)$, since $0 \leq 0$. So it will be enough to show that $\forall x(x \leq 0 \wedge x \neq 0 \equiv x \neq x)$, since then $N x:(x \leq 0 \wedge x \neq 0)=N x:(x \neq x)=0$, , by Log and the definition of zero. Obviously, right-to-left is trivial. So suppose $x \leq 0$ and $x \neq 0$. Then $x<0$. By the roll-back theorem, for some $y, P y 0$ and $0 \leq y$, contradicting axiom (5).

For (ii), suppose the antecedent. Since $n \leq n$,

$$
P(N x:(x \leq n \wedge x \neq n), N x: x \leq n),
$$

by Grundgesetze 102, so it will be enough to show that $\forall x(x \leq n \wedge x \neq n \equiv$ $x \leq m)$. For, if so, then, by $\log , N x:(x \leq n \wedge x \neq n)=N x:(x \leq m)$. But since $P(m, N x: x \leq m)$ and $P m n, N x: x \leq m=n$, by axiom (3). So $N x:(x \leq n \wedge x \neq n)=n$, whence $P(n, N x: x \leq n)$.

which is easily derived from the definition of the ancestral: If $F a$, then, since $\forall x \forall y(F x \wedge$ $R x y \rightarrow F y)$, certainly $\forall x(R a x \rightarrow F x)$. Note that this proof uses no comprehension. 
Left-to-right: Suppose that $x \leq n$ and $x \neq n$. Then $x<n$. By the rollback theorem again, for some $y, P y n$ and $x \leq y$. But Pmn, so, by axiom (4), $y=m$, and so $x \leq m$.

Right-to-left: Suppose that $x \leq m$. By the transitivity of the ancestral, $x \leq n$. So we need only show that $x \neq n$. Suppose that $x=n$. Then $n \leq m$ and Pmn, so, by transitivity again, $m<m$, which contradicts Grundgesetze 145.

\section{Re-Formulating the Prinicples Used In the Usual Proof}

4.1. Three Facts About the Ancestral. The proof just given depends upon three general facts about the ancestral:

Transitivity: $R^{*} a b \wedge R^{*} b c \rightarrow R^{*} a c$

Strong Induction: $R^{*=} a b \wedge F a \wedge \forall x \forall y\left(R^{*=} a x \wedge F x \wedge R x y \rightarrow\right.$

$F y) \rightarrow F b$

The Roll-back Theorem: $R^{*} a b \rightarrow \exists x\left(R x b \wedge R^{*=} a x\right)$

To formulate and prove these results in the present context, we must make suitable assignments of degree to the predicate variables that occur in them.

I shall state the results to be proven below using expressions taken from the language of ramified Frege arithmetic: $0, P,<_{k}$, and $\leq_{k}$, in particular. The proofs of the results, however, do not depend upon any assumptions about these notions other than those explicitly mentioned. So, for example, transitivity (5) in fact holds quite generally, and the restricted form of the roll-back theorem we shall prove below (9) holds for any 'one-many' relation of appropriate degree, that is, for any relation of appropriate degree that satisfies an analogue of $\left(4^{\prime}\right)$. The reader will note that none of these results depend upon HP, and only two of them depend even upon Log.

Transitivity is the easiest of these three facts to handle. Frege's proof, which terminates in proposition 98 of Begriffsschrift [Frege, 1967], requires $\Pi_{1}^{1}$-comprehension, but proposition 98 is in fact provable without any appeal to comprehension [Boolos, 1998d, p. 159]. ${ }^{24}$

Lemma 5. (Transitivity of the Ancestral)

$$
a<_{k} b \wedge b<_{k} c \rightarrow a<_{k} c
$$

Proof. Suppose the antecedent. By definition:

$$
\begin{aligned}
& \text { (i) } \forall F_{k-1}\left[\forall x\left(P a x \rightarrow F_{k-1} x\right) \wedge \forall x \forall y\left(F_{k-1} x \wedge P x y \rightarrow F_{k-1} y\right) \rightarrow\right. \\
& \left.F_{k-1} b\right] \\
& \text { (ii) } \forall F_{k-1}\left[\forall x\left(P b x \rightarrow F_{k-1} x\right) \wedge \forall x \forall y\left(F_{k-1} x \wedge P x y \rightarrow F_{k-1} y\right) \rightarrow\right. \\
& \left.F_{k-1} c\right]
\end{aligned}
$$

\footnotetext{
${ }^{24}$ We will also be using, without special mention: $P a b \rightarrow a \leq_{k} b$. The proof is straightforward, requiring no comprehension: If $P a b$ and $\forall x(P a x \rightarrow F x)$, then certainly $F b$.
} 
Suppose now that $\forall x\left(\operatorname{Pax} \rightarrow F_{k-1} x\right)$ and that $\forall x \forall y\left(F_{k-1} x \wedge P x y \rightarrow F_{k-1} y\right)$. We need to show that $F_{k-1} c$. By (i), $F_{k-1} b$. Since $\forall x \forall y\left(F_{k-1} x \wedge P x y \rightarrow\right.$ $\left.F_{k-1} y\right)$, certainly $\forall x\left(P b x \rightarrow F_{k-1} x\right)$. So $F_{k-1} c$, by (ii).

Strong induction, which Frege proves as Theorem 152 of Grundgesetze, on the other hand, is not predicatively provable. Frege's proof involves applying induction to: $R^{*=} a \xi \wedge F \xi$. Hence, if the initial occurrence of the weak ancestral $R^{*=} a b$ is of degree $k+1$, then this predicate must be of degree $k$, in which case $R^{*=} a \xi$ and $F \xi$ must also be of degree $k$. So the most we should expect to prove is:

Lemma 6. (Strong Induction)

$$
a \leq_{k+1} b \wedge F_{k} a \wedge \forall x \forall y\left(a \leq_{k} x \wedge F_{k} x \wedge P x y \rightarrow F_{k} y\right) \rightarrow F_{k} b
$$

Proof. Suppose the antecedent. Since $a \leq_{k} \xi \wedge F_{k} \xi$ is of degree $k$, we may apply induction to it. We must prove:

(i) $a \leq_{k} a \wedge F_{k} a$

(ii) $a \leq_{k} x \wedge F_{k} x \wedge P x y \rightarrow a \leq_{k} y \wedge F_{k} y$

The former is obvious. The latter follows from transitivity. So $a \leq_{k} b \wedge F_{k} b$, and we are done.

Consider now Frege's proof of the roll-back theorem, which appears as Theorem 142 of Grundgesetze. The proof is by induction, the inductive predicate being: $\exists x\left(R x \xi \wedge R^{*}=a x\right)$. In order for the induction to work, the universally quantified predicate variable occurring in the definition of the initial $R^{*}$ will have to be instantiated with $\exists x\left(R x \xi \wedge R^{*=} a x\right)$. So, if we take $R^{*} a b$ to be of degree $k+1$, then the formula $\exists x\left(R x \xi \wedge R^{*=} a x\right)$ will have to be of degree $k$. Thus, $R^{*=} a x$ has to be of degree $\leq k$. It follows from a notational convention mentioned above that $R$ itself therefore has to be of degree $\leq k$, as well. Thus, Frege's proof will deliver only: $R_{k+1}^{*} a b \rightarrow$ $\exists x\left(R_{k} x b \wedge R_{k}^{*=} a x\right)$.

As it happens, however, we do not need the full force of the roll-back theorem for our purposes here. All we need is $a<_{k} b \rightarrow \exists x\left(P x b \wedge x \leq_{k} b\right)$, and it turns out that this can be derived from $\left(4^{\prime}\right) .{ }^{25}$ First, we prove a more limited result.

Proposition 7. $a<_{k} b \rightarrow \exists x(P x b)$.

\footnotetext{
${ }^{25}$ I owe this observation to an anonymous referee, who pointed out that 7 , Frege's proof of which is also by induction, can be proven by the method used here. The referee then also observed that if we put 7 together with 8 , which I was already using, then we get a version of the roll-back theorem. This observation substantially simplifed the proof of the main result, eliminating the need to appeal to the result mentioned in note 19. As a result, the degree on $\mathbb{N}$ in theorem 18 was reduced from six to two, which is best possible.
} 
Proof. Suppose that $a<_{k} b$ and, for reductio, that $\neg \exists x(P x b)$, which is equivalent to $\forall x \forall y(P x y \rightarrow y \neq b)$. Then by the definition of $<_{k}$ :

$$
\forall F_{k-1}\left(\forall x\left(P a x \rightarrow F_{k-1} x\right) \wedge \forall x \forall y\left(F_{k-1} x \wedge P x y \rightarrow F_{k-1} y\right) \rightarrow F_{k-1} b\right) .
$$

Take $F_{k-1} \xi$ to be: $\xi \neq b$, which is certainly of degree $k-1$. Then we have:

$$
\forall x(P a x \rightarrow x \neq b) \wedge \forall x \forall y(x \neq b \wedge P x y \rightarrow y \neq b) \rightarrow b \neq b .
$$

The two conjuncts in the antecedent then follow from $\forall x \forall y(P x y \rightarrow y \neq b)$, so $b \neq b$. Contradiction.

We now prove a variant of proposition 124 of Frege's Begriffsschrift, which also occurs as Theorem 242 of Grundgesetze:

$$
\forall x \forall y \forall z(R x y \wedge R x z \rightarrow y=z) \wedge R^{*} a b \wedge R a m \rightarrow R^{*=} m b .
$$

The variant we need is:

$$
\forall x \forall y \forall z(P x z \wedge P y z \rightarrow x=y) \wedge a<_{k} b \wedge P m b \rightarrow a \leq_{k} m,
$$

which Frege proves as Theorem 143 of Grundgesetze. ${ }^{26}$ Frege's proof appeals to the roll-back theorem: If $a<_{k} b$, then for some $x, P x b$ and $a \leq_{k} x$. But then $x=m$, since $P m b$, so we are done. We do not have the roll-back theorem available and, indeed, are presently trying to prove a restricted form of it. But it turns out that Theorem 143 can be proven using only predicative comprehension.

Proposition 8. (Grundgesetze 143)

$$
\left(4^{\prime}\right) \vdash a<_{k} b \wedge P m b \rightarrow a \leq_{k} m
$$

Proof. If $a=m$, then $a \leq_{k} m$ trivially, so suppose that $a \neq m$. Suppose further that $P m b$ and, for reductio, that $\neg a \leq_{k} m$, from which it of course follows that $\neg a<_{k} m$. We want to show that $\neg a<_{k} b .^{27}$

Since $\neg a<_{k} m$, we have that, for some $F_{k-1}, \forall x\left(\operatorname{Pax} \rightarrow F_{k-1} x\right), \forall x \forall y\left(F_{k-1} x \wedge\right.$ $P x y \rightarrow F_{k-1} y$ ), and $\neg F_{k-1} m$. We want to show that for some $G_{k-1}$ :
(a) $\forall x\left(P a x \rightarrow G_{k-1} x\right)$
(b) $\forall x \forall y\left(G_{k-1} x \wedge P x y \rightarrow G_{k-1} y\right)$
(c) $\neg G_{k-1} b$

\footnotetext{
${ }^{26}$ Actually, Theorem 143 is just: $a<_{k} b \wedge R m b \rightarrow a \leq_{k} m$. The conjunct $\forall x \forall y \forall z(P x z \wedge$ $P y z \rightarrow x=y$ ) is eliminated slightly earlier, since it is just Theorem 88 .

${ }^{27}$ What follows is not pretty, but the idea is quite simple: If there is an inductive property that all successors of $a$ have that $m$ does not have, then there is no good reason that $b$ should have it, since $m$ is the only predecessor of $b$. (If $b$ had other predecessors, it could be that every inductive property that all successors of $a$ possessed was also possessed by one of them.)
} 
That will imply that $\neg a<_{k} b$. We take $G_{k-1}$ to be: $F_{k-1} \xi \wedge \xi \neq b$, which is clearly of degree $k-1$.

For (a): If $\operatorname{Pax}$, then, since $\forall x\left(\operatorname{Pax} \rightarrow F_{k-1} x\right), F_{k-1} x$. Suppose $x=b$. Then $P a b$ and $P m b$, whence $a=m$, by $\left(4^{\prime}\right)$. But $a \neq m$, so $x \neq b$, and thus $F_{k-1} x \wedge x \neq b$, that is, $G_{k-1} x$.

For (b): Suppose $G_{k-1} x$ - that is, $F_{k-1} x \wedge x \neq b$-and $P x y$. Then certainly $F_{k-1} y$, by heredity. And since $F_{k-1} x$ and $\neg F_{k-1} m, x \neq m$. Suppose $y=b$. Then $P x b$ and $P m b$, so $x=m$, by $\left(4^{\prime}\right)$. So $y \neq b$ and thus $F_{k-1} y \wedge y \neq b$, that is, $G_{k-1} y$.

For (c): Obviously, $\neg\left(F_{k-1} b \wedge b \neq b\right)$, that is, $G_{k-1} b$.

A restricted form of the roll-back theorem now follows quickly.

Lemma 9. $\left(4^{\prime}\right) \vdash a<_{k} b \rightarrow \exists x\left(P x b \wedge a \leq_{k} x\right)$

Proof. Suppose the antecedent. By 7, Pmb, for some $m$. By 8, $a \leq_{k} m$.

And we can now prove the axiom $x \neq 0 \rightarrow \exists y(x=S y)$ of $\mathrm{Q}$, for numbers of any degree:

Corollary 10. $\left(4^{\prime}\right) \vdash \mathbb{N}_{k} n \wedge n \neq 0 \rightarrow \exists m\left(\mathbb{N}_{k} m \wedge P m n\right)$

Proof. Suppose the antecedent. By definition, $0 \leq_{k} n$ and so $0<_{k} n$. So by 9, for some $m$, Pmn and $0 \leq_{k} m$, that is, $\mathbb{N}_{k} m$ and Pmn.

4.2. Grundgesetze 102. The need to reformulate strong induction makes a straightforward adaptation of the standard proof that every natural number has a successor impossible: One cannot simply sprinkle degree subscripts throughout that proof and expect to get anything useful. That is not terribly surprising, since it is not terribly surprising that strong induction is not predicatively provable. What is surprising is that the simple move that is made in the standard proof when one writes

Since $n \leq n, P(N x:(x \leq n \wedge x \neq n), N x: x \leq n)$, by Grundgesetze 102 .

also poses a serious problem. We can easily prove ${ }^{28}$

$$
F_{0} y \rightarrow P\left(N x:\left(F_{0} x \wedge x \neq y\right), N x: F_{0} x\right),
$$

but no matter what degree we assign to $\leq$, it will never be of degree zero. To use Grundgesetze 102 to prove that $P\left(N x:\left(x \leq_{k} n \wedge x \neq n\right), N x: x \leq_{k} n\right)$, that is to say, we would need to prove that, for some $F_{0}$ of degree zero, $\forall x\left[F_{0} x \equiv x \leq_{k} n\right]$. That is not likely to be provable, in general, and messing with the definition of predecession doesn't seem to help. ${ }^{29}$ Fortunately,

\footnotetext{
${ }^{28}$ Or, in the functor formulation: $F_{0} y \wedge \forall x\left[G_{0} x \equiv\left(F_{0} x \wedge x \neq y\right)\right] \rightarrow P\left(\# G_{0}, \# F_{0}\right)$.

${ }^{29} \mathrm{I}$ 'm not absolutely sure it won't help, but my limited experimentation suggests that it just pushes up the degree of $\leq$.
} 
it turns out that we actually only need the following very special case of Grundgesetze 102:

$$
0 \leq_{2} n \wedge a \leq_{1} n \rightarrow P\left(N x:\left(x \leq_{1} n \wedge x \neq a\right), N x: x \leq_{1} n\right) .
$$

And we would have that if we had the following limited form of reducibility:

$$
0 \leq_{2} n \rightarrow \exists F_{0} \forall x\left[F_{0} x \equiv x \leq_{1} n\right],
$$

which can be derived from $\left(4^{\prime}\right)$ and $\left(5^{\prime}\right)$.

We begin by proving two simple facts that we shall need several times.

Fact 11. $\left(5^{\prime}\right) \vdash \neg\left(x<_{k} 0\right)$

Proof. Suppose $x<_{k} 0$. Then 7 implies that for some $y, P y 0$, which contra$\operatorname{dicts}\left(5^{\prime}\right)$.

Corollary 12. $\left(5^{\prime}\right) \vdash \forall x\left(x \leq_{k} 0 \equiv x=0\right)$

Proof. If $x \leq_{k} 0$, then either $x<_{k} 0$ or $x=0$, but the former is ruled out by 11.

Lemma 13. $\left(4^{\prime}\right),\left(5^{\prime}\right) \vdash 0 \leq_{2} n \rightarrow \exists F_{0} \forall x\left[F_{0} x \equiv x \leq_{1} n\right]^{30}$

Proof. The proof is by strong induction on the predicate: $\exists F_{0} \forall x\left[F_{0} x \equiv x \leq_{1}\right.$ $\xi$ ]. The induction is legitimate, because this predicate is of degree 1 . So we must prove:

$$
\begin{aligned}
& \text { (i) } \exists F_{0} \forall x\left[F_{0} x \equiv x \leq_{1} 0\right] \\
& \text { (ii) } 0 \leq_{1} m \wedge \exists F_{0} \forall x\left[F_{0} x \equiv x \leq_{1} m\right] \wedge P m n \rightarrow \exists F_{0} \forall x\left[F_{0} x \equiv\right. \\
& \left.x \leq_{1} n\right]
\end{aligned}
$$

For (i), we may simply take $F_{0} \xi$ to be: $\xi=0$, by 12 .

For (ii), suppose that $0 \leq_{1} m, \forall x\left[G_{0} x \equiv x \leq_{1} m\right]$, and Pmn. It will be enough to show that

$$
\forall x\left[\left(x \leq_{1} m \vee x=n\right) \equiv x \leq_{1} n\right] .
$$

If so, then $\forall x\left[\left(G_{0} x \vee x=n\right) \equiv x \leq_{1} n\right)$, and the formula on the left-hand side is of degree 0 , whence $\exists F_{0} \forall x\left[F_{0} x \equiv x \leq_{1} n\right]$.

Left-to-right: If $x=n$, then $x \leq_{1} n$, trivially; if $x \leq_{1} m$, then, since Pmn, we have $x \leq_{1} n$ again, by transitivity.

Right-to-left: Suppose $x \leq_{1} n$ and $x \neq n$. Then $x<_{1} n$ and so, by 9 , there is some $y$ such that $P y n$ and $x \leq_{1} y$. By $\left(4^{\prime}\right), y=m$, so $x \leq_{1} m$.

\footnotetext{
${ }^{30}$ Here and below, such results are formulated with the minimum degrees they can be. Other forms will also be provable by 'lifting' the degrees. For example, in this case, we have: $\left(4^{\prime}\right),\left(5^{\prime}\right) \vdash 0 \leq_{k+1} n \rightarrow \exists F_{0} \forall x\left[F_{0} x \equiv x \leq_{k} n\right]$, for each $k$.
} 
Corollary 14. Log, $\left(4^{\prime}\right),\left(5^{\prime}\right) \vdash 0 \leq_{2} n \wedge a \leq_{1} n \rightarrow P\left(N x:\left(x \leq_{1} n \wedge x \neq\right.\right.$ a), $\left.N x: x \leq_{1} n\right)^{31}$

Proof. Suppose the antecedent. By 13, for some $F_{0}, F_{0} x \equiv x \leq_{1} n$. Since $a \leq_{1} n, F_{0} a$. Moreover, by Log, $N x: F_{0} x=N x: x \leq_{1} n$. Further: $\left(F_{0} x \wedge x \neq\right.$ $a) \equiv\left(x \leq_{1} n \wedge x \neq a\right)$, whence Log also yields: $N x:\left(F_{0} x \wedge x \neq a\right)=N x$ : $\left(x \leq_{1} n \wedge x \neq a\right)$. That is:

$$
N x: F_{0} x=N x: x \leq_{1} n \wedge F_{0} a \wedge N x:\left(F_{0} x \wedge x \neq a\right)=N x:\left(x \leq_{1} n \wedge x \neq a\right) .
$$

Generalizing on $F_{0}$ and $a$ and applying the definition of $P$ then yields the consequent.

\section{A Predicative Proof That Every Number Has a Successor}

We are now ready to prove that every number has a successor.

We begin with the proof of Theorem 145 of Grundgesetze: $0 \leq n \rightarrow \neg n<$ $n$. One might reasonably have supposed that the proof that every natural number has a successor would require that we have the same subscript both times: Surely $0 \leq_{2} n \rightarrow \neg n<_{1} n$, for example, would not be enough. As it happens, though, it is enough-and it is, in fact, stronger than $0 \leq_{2} n \rightarrow$ $\neg n<_{2} n$, since, as noted above, if $x<_{2} y$, then $x<_{1} y$, so if $\neg n<_{1} n$, then $\neg n<_{2} n$.

Theorem 15. (Grundgesetze 145)

$$
\left(4^{\prime}\right),\left(5^{\prime}\right) \vdash 0 \leq_{2} n \rightarrow \neg n<_{1} n
$$

Proof. Suppose that $0 \leq_{2} n$. Since $\neg n<_{1} n$ is of degree 1, we may apply strong induction. So we must show:

$$
\begin{aligned}
& \text { (i) } \neg 0<{ }_{1} 0 \\
& \text { (ii) } 0 \leq \leq_{1} m \wedge \neg m<{ }_{1} m \wedge P m n \rightarrow \neg n<_{1} n
\end{aligned}
$$

We have already established (i). For (ii), suppose $0 \leq_{1} m, P m n$, and further that $n<_{1} n$. We want to show that $m<_{1} m$. By 9, for some $y, P y n$ and $n \leq_{1} y$. Since Pmn, $y=m$, and so $n<_{1} m$. But also Pmn, so, by transitivity, $m<1 m$.

\footnotetext{
${ }^{31}$ Expressions of the form $N x: A(x)$ have not occurred in any of the proofs to this point - as mentioned above, the results proved so far are all general results about relations satisfying various conditions - so they all go through unchanged if we use the functor $\# F_{0}$, instead, and the alternate definition of $P$ given in note 16 .

Such expressions do occur, however, in the statement of this result, so we need to reformulate it as:

$\log ,\left(4^{\prime}\right),\left(5^{\prime}\right) \vdash 0 \leq_{2} n \wedge a \leq_{1} n \rightarrow \exists F_{0} \exists G_{0}\left[\forall x\left(G_{0} x \equiv x \leq_{1} n\right) \wedge \forall x\left(F_{0} x \equiv\left(x \leq_{1} n \wedge x \neq a\right)\right) \wedge P\left(\# F_{0}, \# G_{0}\right)\right]$

That there is a $G_{0}$ such that $\forall x\left(G_{0} x \equiv x \leq_{1} n\right)$ follows from 13; that there is an $F_{0}$ such that $\forall x\left[F_{0} x \equiv\left(G_{0} x \wedge x \neq n\right)\right]$ and so $\forall x\left[F_{0} x \equiv\left(x \leq_{1} n \wedge x \neq a\right)\right]$ then follows from comprehension, and we can then apply the result mentioned in note 28 to conclude that $P\left(\# F_{0}, \# G_{0}\right)$.
} 
Finally, then, we prove the existence of successors.

Theorem 16. (Grundgesetze 155) ${ }^{32}$

$$
\log ,\left(3^{\prime}\right),\left(4^{\prime}\right),\left(5^{\prime}\right) \vdash 0 \leq_{2} n \rightarrow P\left(n, N x: x \leq_{1} n\right)
$$

Proof. The proof is by strong induction on the predicate: $P\left(\xi, N x: x \leq_{1} \xi\right)$. So we must prove:

(i) $P\left(0, N x: x \leq_{1} 0\right)$

(ii) $0 \leq_{1} m \wedge P\left(m, N x: x \leq_{1} m\right) \wedge P m n \rightarrow P\left(n, N x: x \leq_{1} n\right)$

For (i), by 14, $P\left(N x:\left(x \leq_{1} 0 \wedge x \neq 0\right), N x: x \leq_{1} 0\right)$, since certainly $0 \leq_{1} 0$ and $0 \leq{ }_{2} 0$. Now, by $12, \forall x\left(x \leq_{1} 0 \equiv x=0\right)$, so $\forall x\left[\left(x \leq_{1} 0 \wedge x \neq 0\right) \equiv x \neq x\right]$, whence $N x:\left(x \leq{ }_{1} 0 \wedge x \neq 0\right)=N x:(x \neq x)=0$, by Log and the definition of zero.

For (ii), suppose the antecedent. By $\left(3^{\prime}\right), n=N x: x \leq_{1} m$, so we need only prove

$$
P\left(N x: x \leq_{1} m, N x: x \leq_{1} n\right) .
$$

Since, $0 \leq_{2} m$ and Pmn, $0 \leq_{2} n$, and obviously $n \leq_{1} n$, so we can apply 14 to get:

$$
P\left(N x:\left(x \leq_{1} n \wedge x \neq n\right), N x: x \leq_{1} n\right) .
$$

It will therefore be enough to show that

$$
\forall x\left[\left(x \leq_{1} n \wedge x \neq n\right) \equiv x \leq_{1} m\right],
$$

since then $N x:\left(x \leq_{1} n \wedge x \neq n\right)=N x:\left(x \leq_{1} m\right)$, by Log.

Right-to-left: Suppose that $x \leq_{1} m$. Since Pmn, $x \leq_{1} n$, by transitivity. So we need only show that $x \neq n$. Suppose that $x=n$. Then $n \leq_{1} m$ and Pmn, so, by transitivity again, $m<_{1} m$, which contradicts 15 .

Left-to-right: Suppose that $x \leq_{1} n$ and $x \neq n$. Then $x<_{1} n$. By 9, for some $y, P y n$ and $x \leq_{1} y$. But Pmn, so by $\left(4^{\prime}\right), y=m$, and so $x \leq_{1} m$.

Corollary 17. $0 \leq_{2} n \rightarrow \exists x\left(0 \leq_{2} x \wedge P n x\right)^{33}$

Proof. By the theorem, $P\left(n, N x: x \leq_{1} n\right)$. By transitivity, $0 \leq_{2} N x:\left(x \leq_{1}\right.$ $n)$.

${ }^{32}$ This result can be reformulated as:

$$
\log ,\left(3^{\prime}\right),\left(4^{\prime}\right),\left(5^{\prime}\right) \vdash 0 \leq_{2} n \rightarrow \exists F_{0}\left[\forall x\left(F_{0} x \equiv x \leq_{1} n\right) \wedge P\left(n, \# F_{0}\right)\right] .
$$

The existence of an appropriate $F_{0}$ follows from 13, and the rest of the proof goes through pretty much unchanged.

${ }^{33}$ Obviously, this also follows from the functor formulation of 16 mentioned in note 32 . 


\section{Closing}

We now complete the proof that Robinson arithmetic can be interpreted in ramified Frege arithmetic.

Theorem 18. The following

(1) $\mathbb{N}_{2} 0$

(2) $\mathbb{N}_{2} x \wedge P x y \rightarrow \mathbb{N}_{2} y$

(3) $\forall x \forall y \forall z\left(\mathbb{N}_{2} x \wedge \mathbb{N}_{2} y \wedge \mathbb{N}_{2} z \wedge P x y \wedge P x z \rightarrow y=z\right)$

(4) $\forall x \forall y \forall z\left(\mathbb{N}_{2} x \wedge \mathbb{N}_{2} y \wedge \mathbb{N}_{2} z \wedge P x z \wedge P y z \rightarrow x=y\right)$

(5) $\neg \exists x\left(\mathbb{N}_{2} x \wedge P x 0\right)$

(6) $\forall x\left[\mathbb{N}_{2} x \rightarrow \exists y\left(\mathbb{N}_{2} y \wedge P x y\right)\right]$

(7) $\forall x\left[\mathbb{N}_{2} x \wedge x \neq 0 \rightarrow \exists y\left(\mathbb{N}_{2} y \wedge P y x\right)\right]$

are provable in ramified Frege arithmetic.

Proof. (1) follows immediately from the definition of $\mathbb{N}_{2}$; (2), from transitivity. As we saw earlier, (3), (4), and (5) can be proven purely predicatively, even without the restrictions on the quantifiers. Axiom (6) is corollary 17, and axiom (7) is corollary 10.

Corollary 19. Robinson arithmetic is intepretable in ramified Frege arithmetic.

Proof. As noted earlier, Q can be interpreted in the ramified theory of successor. So we need only show that the ramified theory of successor can be interpreted in ramified Frege arithmetic. Take the domain to be given by $\mathbb{N}_{2} x$. By (1), zero is in it. The successor of anything in the domain then exists by (6), is in the domain by (2), and is unique by (3). That then allows us to define $S x$ as $t y(P x y)$, which we eliminate via Russell's theory of descriptions. We then have $0 \neq S x$ from (5); $S x=S y \rightarrow x=y$, from (4); and $x \neq 0 \rightarrow \exists y(x=S y)$, from (7).

Corollary 20. I $\Delta_{0}(\exp )$ is interpretable in ramified Frege arithmetic.

Proof. By the results of Burgess and Hazen mentioned above.

The situation is thus as follows: Ramified Frege arithmetic interprets $I \Delta_{0}(\exp )$. Frege arithmetic with $\Pi_{1}^{1}$ comprehension interprets PA with $\Pi_{1}^{1}$ induction. What lies in the vast space between these two theories? What forms of reducibility, or other principles we might add to ramified Frege arithmetic, would allow us to interpret intermediate theories? ${ }^{34}$

\footnotetext{
${ }^{34}$ The only result along these lines that is known to me is Fernando Ferreira's proof that, if we add a form of 'finite reducibility' to the ramified predicative fragment of Grundgesetze, which otherwise interprets just $I \Delta_{0}(\exp )$, then we can interpret PA [Ferreira, 2005].
} 


\section{REFERENCES}

[Boolos, 1998a] Boolos, G. (1998a). Frege's theorem and the Peano postulates. In [Boolos, 1998b], (pp. 291-300).

[Boolos, 1998b] Boolos, G. (1998b). Logic, Logic, and Logic. Cambridge MA: Harvard University Press.

[Boolos, 1998c] Boolos, G. (1998c). On the proof of Frege's theorem. In [Boolos, 1998b], (pp. 275-91).

[Boolos, 1998d] Boolos, G. (1998d). Reading the Begriffsschrift. In [Boolos, 1998b], (pp. 155-70).

[Boolos \& Heck, 1998] Boolos, G. \& Heck, R. G. (1998). Die Grundlagen der Arithmetik $\S \S 82-83$. In [Boolos, 1998b], (pp. 315-38).

[Burgess, 1998] Burgess, J. P. (1998). On a consistent subsystem of Frege's Grundgesetze. Notre Dame Journal of Formal Logic, 39, 274-278.

[Burgess, 2005] Burgess, J. P. (2005). Fixing Frege. Princeton NJ: Princeton University Press.

[Burgess \& Hazen, 1998] Burgess, J. P. \& Hazen, A. (1998). Arithmetic and predicative logic. Notre Dame Journal of Formal Logic, 39, 1-17.

[Dedekind, 1902] Dedekind, R. (1902). The nature and meaning of numbers. In Essays on the theory of numbers (pp. 31-115). Chicago: The Open Court Publishing Company.

[Demopoulos, 1995] Demopoulos, W., Ed. (1995). Frege's Philosophy of Mathematics. Cambridge MA: Harvard University Press.

[Ferreira, 2005] Ferreira, F. (2005). Amending Frege's Grundgesetze der Arithmetik. Synthese, 147, 3-19.

[Frege, 1967] Frege, G. (1967). Begriffsschrift: A formula language modeled upon that of arithmetic, for pure thought. In J. van Heijenoort (Ed.), From Frege to Gödel: A Sourcebook in Mathematical Logic (pp. 5-82). Cambridge MA: Harvard University Press.

[Hájek \& Pudlák, 1993] Hájek, P. \& Pudlák, P. (1993). Metamathematics of First-order Arithmetic. New York: Springer-Verlag.

[Heck, 1993] Heck, R. G. (1993). The development of arithmetic in Frege's Grundgesetze der Arithmetik. Journal of Symbolic Logic, 58, 579-601.

[Heck, 1995] Heck, R. G. (1995). Definition by induction in Frege's Grundgesetze der Arithmetik. In [Demopoulos, 1995], (pp. 295-333).

[Heck, 1997a] Heck, R. G. (1997a). Finitude and Hume's Principle. Journal of Philosophical Logic, 26, 589-617.

[Heck, 1997b] Heck, R. G. (1997b). The Julius Caesar objection. In R. Heck (Ed.), Language, Thought, and Logic: Essays in Honour of Michael Dummett (pp. 273-308). Oxford: Clarendon Press.

[Heck, 2000] Heck, R. G. (2000). Cardinality, counting, and equinumerosity. Notre Dame Journal of Formal Logic, 41, 187-209.

[Linnebo, 2004] Linnebo, Ø. (2004). Predicative fragments of Frege arithmetic. Bulletin of Symbolic Logic, 10, 153-74.

It is therefore natural to ask whether Ferreira's result extends to ramified Frege arithmetic. I do not know the answer to this question. It can be shown that the result of adding $0 \leq_{1} x \rightarrow 0 \leq_{2} x$ to ramified FA interprets PA. I have not yet been able to determine the strength of this system, however-though I believe it will prove to be interpretable in $\mathrm{ACA}_{0}$. 
[Wright, 1983] Wright, C. (1983). Frege's Conception of Numbers as Objects. Aberdeen: Aberdeen University Press.

Current address: Department of Philosophy, Brown University, Box 1918, Providence RI 02912, USA 